\title{
Fuzzy models of linear logic
}

\author{
Michael Barr* \\ Department of Mathematics and Statistics \\ McGill University \\ Montreal, Quebec, Canada \\ Email: barr@math.mcgill.ca
}

1999-06-11

\section{Introduction}

There has long been a perception among fuzzy set theorists that the negation based on the operation $a \mapsto 1-a$ is the "correct" negation. It is easy to see why this is so. In the model of fuzzy sets based on the unit interval $[0,1]$, the classical complement of a subset is a destructive operation. For example, if $A_{0}$ is a fuzzy subset of a crisp set $A$, then in the classical complement of $A_{0}$ every element has degree of membership either 0 or 1 . Any element that has any positive degree of membership in $A_{0}$ is entirely out of the complement. This operation is simply too destructive to be useful. It turns out that if the logic is weakened to be linear that the "linear" negation based on $a \mapsto 1-a$ works. As is expected in linear logic the model uses not the category of morphisms, but of relations.

A word about notation. We follow the practice, standard in computer science, of denoting the composite of arrows $f: X \longrightarrow Y$ and $g: Y \longrightarrow Z$ by $f ; g: X \longrightarrow Z$. In order to avoid excess parentheses, we also put functions to the right of the argument, also delimited by semicolons, so that we write $x ; f ; g$ instead of $(f ; g)(x)$. This is based on the perception that there is no difference between thinking of elements of $x \in X$ and functions $x: 1 \longrightarrow X$. Thus $x ; f ; g$ can be read indifferently as the element $x$ to which the composite $f ; g$ is applied or the element $x ; f$ to which the function $g$ is applied. Of course, this notation is abandoned for binary operations that it is customary to infix.

\footnotetext{
* In the preparation of this paper, I have been assisted by a grant from the NSERC of Canada and one from the FCAR of Quebec.
} 


\section{Lattice models}

Let $L$ be a complete $*$-autonomous lattice. This means that $L$ is a complete lattice (whose top and bottom we denote by 1 and 0 , respectively) that is also a $*$-autonomous category. (See [Barr, 1979] or [Barr, 1991] for explanation of $*$-autonomous categories.) That is, there are objects $\top$ and $\perp$ and operations $\otimes, \oplus,(-)^{\perp}$ such that $(\otimes, \top)$ and $(\oplus, \perp)$ are symmetric monoidal structures, $(-)^{\perp}$ is an anti-involution of the order structure that interchanges $\otimes$ and $\oplus$ and interchanges $T$ and $\perp$. In addition we require that

$$
a \otimes b \leq c \Longleftrightarrow a \leq b^{\perp} \oplus c
$$

Note that saying that $\otimes$ and $\oplus$ are monoidal structures implies that they are covariant functors, that is order preserving.

2.1 Example. An example of such a structure is given by letting $L$ be the unit interval $[0,1]$ with $\top=1, \perp=0, a \oplus b=\min (a+b, 1), a \otimes b=\max (a+b-1,0)$ and $a^{\perp}=1-a$. Other examples using the same operations include the set of rationals in the unit interval and the finite sets of all rationals with a fixed denominator. Since an arbitrary cartesian products of these lattices in again one, we can construct many others that are not total orders.

2.2 Example. Here is another class of examples in which (with one exceptional case) the four constants $0,1, \top$ and $\perp$ are all different, as are the four binary operations + , $\times, \otimes$ and $\oplus$. Take $L$ to be the two point completion of the real line (so it is again the unit interval, but it is hard to describe the operations if we think of it as that). In order to avoid confusion, we will denote the added elements by $-\infty$ and $\infty$ (instead of 0 and 1). Let $a$ be a real number, which is a parameter. Let $\top=-a$ and $\perp=0$. The operations $\oplus$ and $\otimes$ are given by the following tables, in which $x$ and $y$ stand for finite numbers.

\begin{tabular}{c|ccc}
$\oplus$ & $\infty$ & $-\infty$ & $x$ \\
\hline$\infty$ & $\infty$ & $\infty$ & $\infty$ \\
$-\infty$ & $\infty$ & $-\infty$ & $-\infty$ \\
$y$ & $\infty$ & $\infty$ & $x+y$
\end{tabular}

\begin{tabular}{c|ccc}
$\otimes$ & $\infty$ & $-\infty$ & $x$ \\
\hline$\infty$ & $\infty$ & $-\infty$ & $\infty$ \\
$-\infty$ & $-\infty$ & $-\infty$ & $-\infty$ \\
$y$ & $\infty$ & $-\infty$ & $x+y+a$
\end{tabular}

The case $a=0$ is also interesting. There $\otimes=\oplus$ and $\top=\perp$.

Except for the $\infty$ and $-\infty$, this is the same as the shift monoid recently discovered by Robin Cockett [Cockett \& Seely, to appear].

To show that this is $*$-autonomous, we begin with the semi-distributive identity (see [Cockett \& Seely, to appear]). 
2.3 Proposition. For any $x, y, z \in L$, we have

$$
x \otimes(y \oplus z) \leq(x \otimes y) \oplus z
$$

Proof. We clearly have equality if all three elements are finite. For the rest, it is just a matter of looking at cases. If $x=-\infty$, then the left hand side is $-\infty$ so the inequality is certainly satisfied. Similarly, if $z=\infty$, then the right hand side is $\infty$. If $x=\infty$, then the right hand side is $\infty$ unless $y=-\infty$. In that case, the left hand side is $-\infty$ unless $z=\infty$, a case we have already dealt with. Dually, the inequality is satisfied if $z=-\infty$. This takes care of all cases in which either $x$ or $z$ is infinite. If they are both finite and $z$ is not, then both sides reduce to $z$.

The remaining things, such as that $\otimes$ and $\oplus$ are commutative monoids, that $x^{\perp \perp}=$ $x,(x \otimes y)^{\perp}=x^{\perp} \oplus y^{\perp}, x \otimes x^{\perp} \leq \perp$ and their duals, are trivial. From this we can readily show that $x \otimes y \leq z$ if and only if $x \leq y^{\perp} \oplus z$. In fact, assuming the first, we have

$$
x=\top \otimes x \leq\left(y^{\perp} \oplus y\right) \otimes x \leq y^{\perp} \oplus(y \oplus x) \leq y^{\perp} \oplus z
$$

The other direction is dual.

We note that when $a>0$, we do not have $x \otimes y \leq x \oplus y$, which means the "mix" rule (see [Cockett and Seely, to appear) is not always satisfied.

\section{$3 \quad L$-fuzzy sets}

3.1 The category. If $L$ is a $*$-autonomous lattice, an $L$-fuzzy set is a pair $(X, \xi)$ where $X$ is a set and $\xi: X \longrightarrow L$ is a function. If $(X, \xi)$ and $(Y, \theta)$ are $L$-fuzzy sets, an $L$-fuzzy relation $\phi:(X, \xi) \longrightarrow(Y, \theta)$ is a function $\phi: X \times Y \longrightarrow L$ such that $(x, y) ; \phi \leq$ $(x ; \xi)^{\perp} \oplus y ; \theta$. This condition is equivalent to the assumption that $x ; \xi \otimes(x, y) ; \phi \leq y ; \theta$.

We compose arrows as follows. If $\phi:(X, \xi) \longrightarrow(Y, \theta)$ and $\gamma:(Y, \theta) \longrightarrow(Z, \zeta)$ are arrows, we let $\phi ; \gamma:(X, \xi) \longrightarrow(Z, \zeta)$ by

$$
(x, z) ; \phi ; \gamma=\bigvee_{y \in Y}(x, y) ; \phi \otimes(y, z) ; \gamma
$$

We have for any $x \in X, y \in Y$ and $z \in Z$,

$$
x ; \xi \otimes(x, y) ; \phi \otimes(y, z) ; \gamma \leq y ; \theta \otimes(y, z) ; \gamma \leq z ; \zeta
$$

whence $(x, y) ; \phi \otimes(y, z) ; \gamma \leq(x ; \xi)^{\perp} \oplus z ; \zeta$. Taking the sup over all $y$ we conclude that

$$
(x, z) ; \phi ; \gamma \leq(x ; \xi)^{\perp} \oplus z ; \zeta
$$


We show associativity of composition as follows. Since $-\otimes b$, is left adjoint to $-\oplus b^{\perp}$, it preserves sup. Then for $\eta:(Z, \zeta) \longrightarrow(T, \tau), x \in X$ and $t \in T$,

$$
\begin{aligned}
(x, t) ;(\phi ; \gamma) ; \eta & =\bigvee_{z \in Z}(x, z) ; \phi ; \gamma \otimes(z, t) ; \eta \\
& =\bigvee_{z \in Z}\left(\bigvee_{y \in Y}(x, y) ; \phi \otimes(y, z) ; \gamma\right) \otimes(z, t) ; \eta \\
& =\bigvee_{z \in Z}\left(\bigvee_{y \in Y}(x, y) ; \phi \otimes(y, z) ; \gamma \otimes(z, t) ; \eta\right) \\
& =\bigvee_{(y, z) \in Y \times Z}(x, y) ; \phi \otimes(y, z) ; \gamma \otimes(z, t) ; \eta
\end{aligned}
$$

The similar computation of $(x, t) ; \phi ;(\gamma ; \eta)$ establishes the associativity of composition. The identity arrow $\iota:(X, \xi) \longrightarrow(X, \xi)$ is defined by

$$
\left(x_{1}, x_{2}\right) ; \iota= \begin{cases}\top, & \text { if } x_{1}=x_{2} \\ 0, & \text { otherwise }\end{cases}
$$

The verification that this is the identity is left to the reader. It requires knowing that $a \otimes \top=a$, which is part of the definition of monoidal categories and that $a \otimes 0=0$ which follows from the fact that $a \otimes-$, being a left adjoint, preserves the bottom.

We denote the resultant category by $\operatorname{Rel}(L)$. If $(X, \xi)$ is an object of this category, we will say that $X$ is its carrier and $\xi$ its structure map.

3.2 Crisp maps. For any set $X$, let $\left(X, \xi_{0}\right)$ denote the object of $\operatorname{Rel}(L)$ given by $x ; \xi_{0}=\top$ for all $x \in X$. This is called the crisp object of $\operatorname{Rel}(L)$ corresponding to $X$. If $f: X \rightarrow Y$ is a function and $\left(Y, \theta_{0}\right)$ is the crisp object corresponding to $Y$, let $f_{0}: X \times Y \longrightarrow L$ be defined by

$$
(x, y) ; f_{0}= \begin{cases}\top, & \text { if } y=x ; f \\ 0, & \text { otherwise }\end{cases}
$$

Then for any $x \in X, x ; \xi \otimes(x, y) ; f_{0} \leq y ; \theta_{0}$ is automatic since the right hand side is T. If $g: Y \longrightarrow Z$ is another function, then for $x \in X$ and $z \in Z$,

$$
(x, z) ; f_{0} ; g_{0}=\bigvee_{y \in Y}(x, y) ; f_{0} \otimes(y, z) ; g_{0}
$$

If $z=x ; f ; g$ there is just one non-0 term in that sup, namely $(x, x ; f) \otimes(x ; f, z)=$ $\top \otimes \top=\top$ and if $z \neq x ; f ; g$ there are none since for all $y \in Y$ at least one of $(x, y) ; f_{0}$ and $(y, z) ; g_{0}$ is 0 . Thus $f_{0} ; g_{0}=(f ; g)_{0}$. We have just seen that $\iota=\mathrm{id}_{0}$ is the identity, so that this construction describes a functor from sets to $L$-fuzzy sets. 
3.3 The duality. There is a functor that we denote by $(-)^{\perp}: \operatorname{Rel}(L)^{\mathrm{op}} \longrightarrow \operatorname{Rel}(L)$ such that $(X, \xi)^{\perp}=\left(X, \xi^{\perp}\right)$ where we let $x ; \xi^{\perp}=(x ; \xi)^{\perp}$. Now $\phi: X \times Y \rightarrow L$ satisfies $(x, y) ; \phi \leq x ; \xi^{\perp} \oplus y ; \theta$ if and only if it also satisfies $(x, y) ; \phi \leq\left(y ; \theta^{\perp}\right)^{\perp} \oplus x ; \xi^{\perp}$. Thus we can define $(y, x) ; \phi^{\perp}=(x, y) ; \phi$. Note that $((x, y) ; \phi)^{\perp}$ does not work.

Hence $(-)^{\perp}$ is a contravariant involution on $\operatorname{Rel}(L)$.

3.4 The tensor and cotensor. Let $(X, \xi)$ and $(Y, \theta)$ be objects of $\operatorname{Rel}(L)$. We define $(X, \xi) \otimes(Y, \theta)=(X \times Y, \xi \otimes \theta)$ where $(x, y) ;(\xi \otimes \theta)=x ; \xi \otimes y ; \theta$. This defines the tensor; the cotensor is defined by duality. This evidently gives that $(X, \xi) \oplus(Y, \theta)=$ $(X \times Y, \xi \oplus \theta)$ where $(x, y) ;(\xi \oplus \theta)=x ; \xi \oplus y ; \theta$.

3.5 Proposition. There is a one-one correspondence between arrows $(X, \xi) \otimes(Y, \theta)$ $\longrightarrow(Z, \zeta)$ and arrows $(X, \xi) \longrightarrow(Y, \theta)^{\perp} \oplus(Z, \zeta)$.

Proof. Both kinds require a function $\phi: X \times Y \times Z \longrightarrow L$. The first one subjects $\phi$ to the condition $(x, y, z) ; \phi \leq(x, y) ;(\xi \otimes \theta)^{\perp} \oplus z ; \zeta$ while the second to the condition $\phi(x, y, z) \leq x ; \xi^{\perp} \oplus y ; \theta^{\perp} \oplus z ; \zeta$ and these are evidently the same.

We have to say how tensor and cotensor act as functors. For tensor, this is as expected. If $\alpha:(X, \xi) \longrightarrow\left(X^{\prime}, \xi^{\prime}\right)$ and $\beta:(Y, \theta) \longrightarrow\left(Y^{\prime}, \theta^{\prime}\right)$ are morphisms, then $\alpha \otimes$ $\beta:(X \times Y, \xi \otimes \theta) \longrightarrow\left(X^{\prime} \times Y^{\prime}, \xi^{\prime} \otimes \theta^{\prime}\right)$ is given by

$$
\left(x, y, x^{\prime}, y^{\prime}\right) ; \alpha \otimes \beta=\left(x, x^{\prime}\right) ; \alpha \otimes\left(y, y^{\prime}\right) ; \beta
$$

Since $\left(x, x^{\prime}\right) ; \alpha \otimes x ; \xi \leq x^{\prime} ; \xi^{\prime}$ and $\left(y, y^{\prime}\right) ; \beta \otimes y ; \theta \leq y^{\prime} ; \theta^{\prime}$, we have

$$
\left(x, y, x^{\prime}, y^{\prime}\right) ;(\alpha \otimes \beta) \otimes(x, y) ;(\xi \otimes \theta)=\left(x, x^{\prime}\right) ; \alpha \otimes\left(y, y^{\prime}\right) ; \beta \otimes x ; \xi \otimes y ; \theta \leq x^{\prime} ; \xi^{\prime} \otimes y^{\prime} ; \theta^{\prime}
$$

so that $\alpha \otimes \beta$ is a morphism. The cotensor is not quite as expected. In fact,

$$
\left(x, y, x^{\prime}, y^{\prime}\right) ;(\alpha \oplus \beta)=\left(x, x^{\prime}\right) ; \alpha \otimes\left(y, y^{\prime}\right) ; \beta
$$

which is exactly the same formula as the tensor. The explanation for this (aside from the fact that the formula with a $\oplus$ on the right hand side does not work) is that we work out the cotensor by dualizing, taking the tensor and dualizing and the dual of an arrow has the same formula as the arrow. Hence the same formula must give both the tensor and cotensor.

The next proof also shows that the tensor and cotensor have to have the same formula.

3.6 Proposition. The correspondence between arrows of Proposition 3.5 is natural in all three arguments. 
Proof. Suppose we have arrows $\alpha:\left(X^{\prime}, \xi^{\prime}\right) \longrightarrow(X, \xi), \beta:\left(Y^{\prime}, \theta^{\prime}\right) \longrightarrow(Y, \theta)$ and $\psi:(Z, \zeta)$ $\longrightarrow\left(Z^{\prime}, \zeta^{\prime}\right)$. We must show that the square

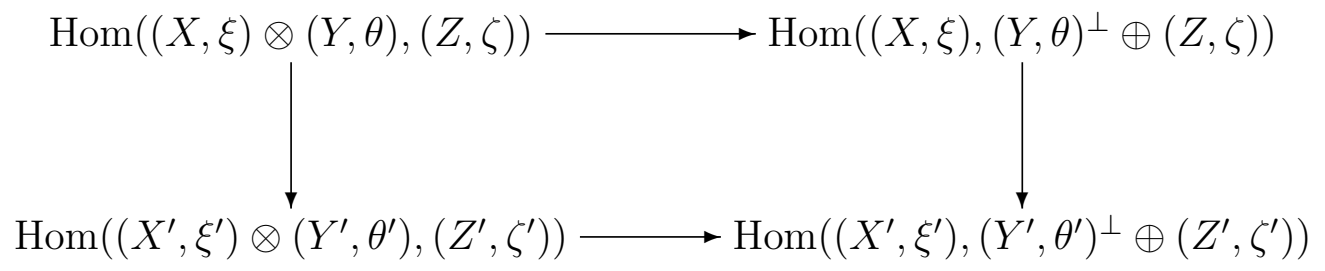

commutes. Assume that $\phi:(X, \xi) \otimes(Y, \theta) \longrightarrow(Z, \zeta))$ is given and $\widehat{\phi}:(X, \xi) \longrightarrow(Y, \theta)^{\perp} \oplus$ $(Z, \zeta)$ is its transpose. We must show that the two composites

$$
\left(X^{\prime}, \xi^{\prime}\right) \otimes\left(Y^{\prime}, \theta^{\prime}\right) \stackrel{\alpha \times \beta}{\longrightarrow}(X, \xi) \times(Y, \theta) \stackrel{\phi}{\longrightarrow}(Z, \zeta) \stackrel{\psi}{\longrightarrow}\left(Z^{\prime}, \zeta^{\prime}\right)
$$

and

$$
\left(X^{\prime}, \xi^{\prime}\right) \stackrel{\alpha}{\longrightarrow}(X, \xi) \stackrel{\widehat{\phi}}{\longrightarrow}(Y, \theta)^{\perp} \oplus(Z, \zeta) \stackrel{\beta^{\perp} \oplus \psi}{\longrightarrow}\left(Y^{\prime}, \theta^{\prime \perp}\right) \oplus\left(Z^{\prime}, \zeta^{\prime}\right)
$$

have the same formula. The first is given by the formula

$$
\left(x^{\prime}, y^{\prime}, z^{\prime}\right) ; \alpha \otimes \beta ; \phi ; \psi=\bigvee_{x, y, z}\left(x^{\prime}, x\right) ; \alpha \otimes\left(y^{\prime}, y\right) ; \beta \otimes(x, y, z) ; \phi \otimes\left(z, z^{\prime}\right) ; \psi
$$

The second is given by

$$
\left(x^{\prime}, y^{\prime}, z^{\prime}\right) ; \alpha ; \widehat{\phi} ; \beta^{\perp} \oplus \psi=\bigvee_{x, y, z}\left(x^{\prime}, x\right) ; \alpha \otimes(x, y, z) ; \widehat{\phi} \otimes\left(y, z, y^{\prime}, z^{\prime}\right) ;\left(\beta^{\perp} \oplus \psi\right)
$$

$\operatorname{But}(x, y, z) ; \widehat{\phi}=(x, y, z) ; \phi$ and

$$
\left(y, z, y^{\prime}, z^{\prime}\right) ;\left(\beta^{\perp} \oplus \psi\right)=\left(y, y^{\prime}\right) ; \beta^{\perp} \otimes\left(z, z^{\prime}\right) ; \psi=\left(y^{\prime}, y\right) ; \beta \otimes\left(z, z^{\prime}\right) ; \psi
$$

so the two formulas are the same.

Thus we have the adjointness required for a $*$-autonomous category. Let $T$ denote the object $(1, T)$, that is the object with one element carrier and the function taking it to $T \in L$ and similarly $\perp$ denote $(1, \perp)$. One sees immediately that $T^{\perp}=\perp$, that $\top$ is a unit for $\otimes$ and $\perp$ is a unit for $\oplus$. Thus these structures give a $*$-autonomous category.

\section{Category-based fuzzy sets}

Following a suggestion of Robin Cockett's we show that there is a very similar construction in which we replace a $*$-autonomous poset by a $*$-autonomous category. 
4.1 The category $\operatorname{Fuz}(\mathscr{A})$. Let $\mathscr{A}$ be a $*$-autonomous category in which every family $\left\{A_{i} \mid i \in I\right\}$ of objects has a sum, which we denote $\sum_{i \in I} A_{i}$. Let $A_{0}$ and $A_{1}$ be the sets of objects and arrows, respectively, of the category underlying $\mathscr{A}$. We are going to define a category $\operatorname{Fuz}(\mathscr{A})$ of $\mathscr{A}$-valued fuzzy sets. Although it is not strictly necessary, we will simplify the exposition by supposing that there is a unique initial object 0 and that the isomorphisms $A \cong A \otimes 0 \cong 0$ and $A \otimes \top \cong \top \otimes A \cong A$ are actual equalities for all objects $A$ of $\mathscr{A}$.

An $\mathscr{A}$-valued fuzzy set consists of a pair $(X, \xi)$ where $X$ is a set and $\xi: X$ $\longrightarrow A_{0}$ is a function. If $(X, \xi)$ and $(Y, \theta)$ are $\mathscr{A}$-valued fuzzy sets, a morphism is an equivalence class of pairs $\left(\phi_{0}, \phi_{1}\right)$ where $\phi_{0}: X \times Y \longrightarrow A_{0}$ and $\phi_{1}: X \times Y \longrightarrow A_{1}$ are functions such that for all $(x, y) \in X \times Y$,

$$
(x, y) ; \phi_{1}: x ; \xi \otimes(x, y) ; \phi_{0} \longrightarrow y ; \theta
$$

We say that $\left(\phi_{0}, \phi_{1}\right)=\left(\phi_{0}^{\prime} \phi_{1}^{\prime}\right)$ if there is an $X \times Y$-indexed family of isomorphisms $(x, y) ; \alpha:(x, y) ; \phi_{0} \longrightarrow(x, y) ; \phi_{0}^{\prime}$ such that for all $x \in X$ and $y \in Y$, the triangle

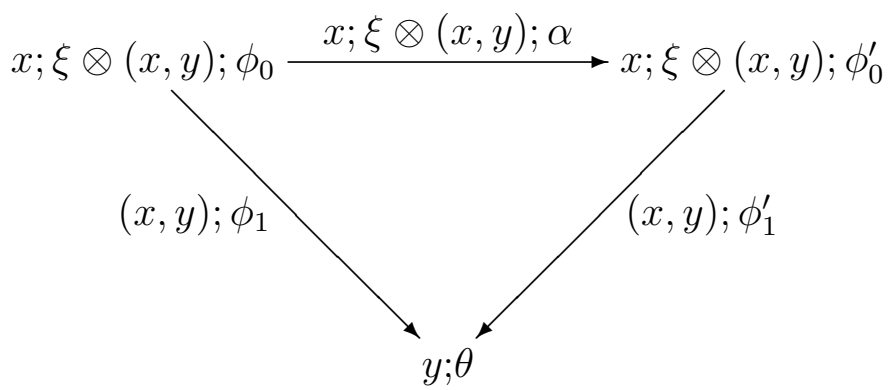

commutes.

The transpose of a morphism $\left(\phi_{0}, \phi_{1}\right)$ is the pair $\left(\phi_{0}, \widehat{\phi}_{1}\right)$ where $\widehat{\phi}_{1}:(x, y) ; \phi_{0} \longrightarrow$ $x ; \xi^{\perp} \oplus y ; \theta$ is the transpose of $\phi_{1}$. Morphisms could be defined in terms of the transpose and both possible definitions of morphism will have their use.

4.2 Composition and identities. Here is how we compose morphisms. This definition could not possibly be associative without the equivalence relation just defined. As a matter of fact, it is not even well defined, given that sums are defined only up to isomorphism. If $\left(\phi_{0}, \phi_{1}\right): X \rightarrow Y$ and $\left(\gamma_{0}, \gamma_{1}\right): Y \rightarrow Z$ are morphisms, we define the composite $\left(\phi_{0}, \phi_{1}\right) ;\left(\gamma_{0}, \gamma_{1}\right)=\left(\eta_{0}, \eta_{1}\right): X \longrightarrow Z$ as follows.

$$
(x, z) ; \eta_{0}=\sum_{y \in Y}(x, y) ; \phi_{0} \otimes(y, z) ; \gamma_{0}
$$

To define $\eta_{1}$ we first note that since $A \otimes-$ has a right adjoint for any object $A$ of $\mathscr{A}$, it commutes with arbitrary sums. Thus,

$$
x ; \xi \otimes(x, z) ; \eta_{0} \cong \sum_{y \in Y}(x, y) ; \phi_{0} \otimes(y, z) ; \gamma_{0}
$$


Let us introduce the nonce notation

$$
\langle\langle x, y, z\rangle\rangle: x ; \xi \otimes(x, y) ; \phi_{0} \otimes(y, z) ; \gamma_{0} \longrightarrow x ; \xi \otimes(x, z) ; \eta_{0}
$$

for the canonical inclusion. Then to define $\eta_{1}$ it is sufficient to define $\langle\langle x, y, z\rangle\rangle ; \eta_{1}$ for all $x, y$ and $z$. We define it as the composite

$$
x ; \xi \otimes(x, y) ; \phi_{0} \otimes(y, z) ; \gamma_{0} \stackrel{(x, y) ; \phi_{1} \otimes \mathrm{id}}{\longrightarrow} y ; \theta \otimes(y, z) ; \gamma_{0} \stackrel{\gamma_{1}}{\longrightarrow} z ; \zeta
$$

as required. To prove associativity, we suppose also a morphism $\left(\kappa_{0}, \kappa_{1}\right):(Z, \zeta) \longrightarrow$ $(W, \omega)$. Then after the distributive isomorphisms of tensor over sum are applied, both composites turn out to the same morphism

$$
\begin{gathered}
x ; \xi \otimes(x, y) ; \phi_{0} \otimes(y, z) ; \gamma_{0} \otimes(z, w) ; \kappa_{0} \stackrel{(x, y) ; \phi_{1} \otimes \mathrm{id} \otimes \mathrm{id}}{\longrightarrow} y ; \theta \otimes(y, z) ; \gamma_{0} \otimes(z, w) \\
\stackrel{\gamma_{1} \otimes \mathrm{id}}{\longrightarrow} z ; \zeta \otimes(z, w) \stackrel{\kappa_{1}}{\longrightarrow}(z, w)
\end{gathered}
$$

The identity morphism of $(X, \xi)$ is given by $\left(\iota_{0}, \iota_{1}\right)$ where

$$
\left(x, x^{\prime}\right) ; \iota_{0}= \begin{cases}\top & \text { if } x=x^{\prime} \\ 0 & \text { otherwise }\end{cases}
$$

Note that this alternates between the tensor unit and the categorical initial object. The reason is that $T$ is the tensor unit and 0 is the tensor zero. It does not mean that they are in any sense operations dual to each other. The morphism $\left(x, x^{\prime}\right) ; \iota_{1}: x ; \xi \otimes\left(x, x^{\prime}\right) ; \iota_{0}$ $\longrightarrow x^{\prime} ; \xi$ is defined as

$$
\left(x, x^{\prime}\right) ; \iota_{1}= \begin{cases}\text { id: } x ; \xi \otimes \top \longrightarrow x ; \xi & \text { if } x=x^{\prime} \\ 0 \longrightarrow x^{\prime} ; \xi & \text { otherwise }\end{cases}
$$

Let us write $\left(\iota_{0}, \iota_{1}\right) ;\left(\phi_{0}, \phi_{1}\right)=\left(\phi_{0}^{\prime}, \phi_{1}^{\prime}\right)$. Then

$$
(x, y) ; \phi_{0}^{\prime}=\sum_{x^{\prime} \in X}\left(x, x^{\prime}\right) ; \iota_{0} \otimes\left(x^{\prime}, y\right) ; \phi_{0}
$$

But $\left(x, x^{\prime}\right)=0$, unless $x=x^{\prime}, 0 \otimes\left(x^{\prime}, y\right) \cong 0$ and 0 is the unit for the categorical sum so that $(x, y) ; \phi_{0}^{\prime} \cong(x, y) ; \phi_{0}$. Moreover, $\phi_{1}^{\prime}$ corresponds under the isomorphism to $\langle\langle x, x, y\rangle\rangle ;(x, y) ; \phi_{0}$ which is

$$
x ; \xi \otimes(x, y) ; \phi_{0} \stackrel{\mathrm{id} \otimes \mathrm{id}}{\longrightarrow} x ; \xi \otimes(x, y) ; \phi_{0} \stackrel{\phi_{1}}{\longrightarrow} y ; \theta
$$

which is $\left(\phi_{0}, \phi_{1}\right)$. The argument on the other side is similar. 
4.3 Crisp morphisms. Just as in the case of $L$-fuzzy sets, there is a natural embedding of functions $X \rightarrow Y$ as morphisms of $\mathbf{F u z}(\mathscr{A})$. If $f: X \rightarrow Y$ is a function, define $f_{0}: X \times Y \longrightarrow A_{0}$ by

$$
(x, y) ; f_{0}= \begin{cases}\top & \text { if } y=x ; f \\ 0 & \text { otherwise }\end{cases}
$$

and $f_{1}: X \times Y \longrightarrow A_{1}$ by

4.4 The *-autonomous structure. Let $(X, \xi)$ and $(Y, \theta)$ be objects of $\mathbf{F u z}(\mathscr{A})$. We define

$$
(X, \xi) \otimes(Y, \theta)=(X \times Y, \xi \otimes \theta)
$$

where $(x, y) ; \xi \otimes \theta=x ; \xi \otimes y \theta$. If $\left(\phi_{0}, \phi_{1}\right):(X, \xi) \longrightarrow\left(X^{\prime}, \xi^{\prime}\right)$ and $\left(\gamma_{0}, \gamma_{1}\right):(Y, \theta) \longrightarrow$ $\left(Y^{\prime}, \theta^{\prime}\right)$ are arrows of $\mathbf{F u z}(\mathscr{A})$, we define

$$
\left(\phi_{0}, \phi_{1}\right) \otimes\left(\gamma_{0}, \gamma_{1}\right)=\left(\phi_{0} \otimes \gamma_{0}, \phi_{1} \otimes \gamma_{1}\right):(X, \xi) \otimes\left(X^{\prime}, \xi^{\prime}\right) \longrightarrow(Y, \theta) \otimes\left(Y^{\prime}, \theta^{\prime}\right)
$$

by the formulas

$$
\begin{aligned}
\left(x, y, x^{\prime}, y^{\prime}\right) ; \phi_{0} \otimes \gamma_{0} & =\left(x, x^{\prime}\right) ; \phi_{0} \otimes\left(y, y^{\prime}\right) ; \gamma_{0} \\
x ; \xi \otimes y ; \theta \otimes\left(x, x^{\prime}\right) ; \phi_{0} \otimes\left(y, y^{\prime}\right) ; \gamma_{0} & \longrightarrow x ; \xi \otimes\left(x, x^{\prime}\right) ; \phi_{0} \otimes y ; \theta \otimes\left(y, y^{\prime}\right) ; \gamma_{0} \\
& \stackrel{\phi_{1} \otimes \gamma_{1}}{\longrightarrow} x^{\prime} ; \xi^{\prime} \otimes y^{\prime} ; \theta^{\prime}
\end{aligned}
$$

The second formula uses the symmetry of the tensor in a non-trivial way and there does not appear to be any way of making this work in the non-symmetric case.

The unit for the tensor is $(1, T)$ where we use $T$ to denote the constant map whose value is $T$. It is evident that this is the unit for the tensor. For an object $(X, \xi)$, the dual object $(X, \xi)^{\perp}=\left(X, \xi^{\perp}\right)$ where $\xi^{\perp}$ is defined by $x ; \xi^{\perp}=(x ; \xi)^{\perp}$. This implies that $(X, \xi) \oplus(Y, \theta)=(X \times Y, \xi \oplus \theta)$. Just as in the case of a poset, the tensor sum of $\left(\phi_{0}, \phi_{1}\right):(X, \xi) \longrightarrow\left(X^{\prime}, \xi^{\prime}\right)$ and $\left(\gamma_{0}, \gamma_{1}\right):(Y, \theta) \longrightarrow\left(Y^{\prime}, \theta^{\prime}\right)$ is given by

$$
\left(x, y, x^{\prime}, y^{\prime}\right) ; \phi_{0} \oplus g=\left(x, x^{\prime}\right) ; f \otimes\left(y, y^{\prime}\right) ; \gamma_{0}
$$

and

$$
\begin{aligned}
& x ; \xi \oplus y ; \theta \otimes\left(x, x^{\prime}\right) ; \phi_{0} \otimes\left(y, y^{\prime}\right) ; \gamma_{0} \longrightarrow x ; \xi \otimes\left(x, x^{\prime}\right) ; \phi_{0} \otimes y ; \theta \otimes\left(y, y^{\prime}\right) ; \gamma_{0} \\
& \stackrel{\phi_{1} \otimes \gamma_{1}}{\longrightarrow} x^{\prime} ; \xi^{\prime} \otimes y^{\prime} ; \theta^{\prime}
\end{aligned}
$$




\section{Completeness}

5.1 Products and sums. In this section we show that the sums in the category $\mathscr{A}$ lead to a construction in $\operatorname{Fuz}(\mathscr{A})$ that is simultaneously the sum and product. Let $\left\{\left(X_{i}, \xi_{i}\right)\right\}$ be a family, which we will suppose disjoint, of objects of $\mathbf{F u z}(\mathscr{A})$. Let $X=\sum X_{i}$ and $\xi: X \rightarrow A_{0}$ be the unique function whose restriction to $X_{i}$ is $\xi_{i}$. We define arrows $\pi^{i}=\left(\pi_{0}^{i}, \pi_{1}^{i}\right):(X, \xi) \longrightarrow\left(X_{i}, \xi_{i}\right)$. For the first, we let

$$
\left(x, x_{i}\right) ; \pi_{0}^{i}=\left\{\begin{array}{cl}
\top & \text { if } x=x_{i} \\
0 & \text { otherwise }
\end{array}\right.
$$

The second is defined by

$$
\pi_{1}^{i}= \begin{cases}\mathrm{id}: x ; \xi \otimes \top \longrightarrow x_{i} ; \xi_{i} & \text { if } x=x_{i} \\ 0 \longrightarrow x_{i} ; \xi_{i} & \text { otherwise }\end{cases}
$$

Suppose we have a family of morphisms $\left(\phi_{0}^{i}, \phi_{1}^{i}\right):(Y, \theta) \longrightarrow\left(X_{i}, \xi_{i}\right)$. Define $\left(\phi_{0}, \phi_{1}\right):(Y, \theta)$ $\longrightarrow(X, \xi)$ as follows. For $x \in X_{i}$ define $(y, x) ; \phi_{0}=(y, x) ; \phi_{0}^{i}$ and

$$
(y, x) ; \phi_{1}=(y, x) ; \phi_{1}^{i}: y ; \theta \otimes(y, x) ; \phi_{0}^{i} \longrightarrow x ; \xi_{0}^{i}
$$

If we write $\left(\phi_{0}, \phi_{1}\right) ;\left(\pi_{0}^{i}, \pi_{1}^{i}\right)=\left(\eta_{0}^{i}, \eta_{1}^{i}\right)$, then for $x \in X_{i}$,

$$
\left(y, x_{i}\right) ; \eta_{0}^{i}=\sum_{x \in X}(y, x) ; \phi_{0} \otimes\left(x, x_{i}\right) \xi_{i}
$$

The second factor of every term in this sum is 0 except the one for which $x=x_{i}$. This term is $(y, x) ; \phi_{0} \otimes \top=\left(y, x_{i}\right) ; \phi_{0}^{i}$, so that $\eta_{0}^{i}=\phi_{0}^{i}$. We need calculate $\left(y, x_{i}\right) ; \eta_{1}^{i}$ on the only possible non-zero summand, namely the unique one for which $x=x_{i}$. On this summand, $\left(y, x_{i}\right) ; \eta_{1}^{i}$ is the composite

$$
y ; \theta_{0} \otimes(y, x) ; \phi_{0} \otimes\left(x, x_{i}\right) ; \pi_{0}^{i} \stackrel{(y, x) ; \phi_{1} \otimes \mathrm{id}}{\longrightarrow} x ; \xi_{0} \otimes\left(x, x_{i}\right) ; \pi_{0}^{i} \stackrel{\pi_{1}^{i}}{\longrightarrow} x_{i} ; \xi_{i}
$$

which is the same as

$$
y ; \theta_{0} \otimes\left(y, x_{i}\right) ; \phi_{0}^{i} \stackrel{\left(y, x_{i}\right) ; \phi_{1}^{i}}{\longrightarrow} x_{i} ; \xi_{0}^{i} \stackrel{\mathrm{id}}{\longrightarrow} x_{i} ; \xi_{i}
$$

so that $\eta_{1}^{i}=\phi_{1}^{i}$.

The uniqueness comes about as follows. Let $\left(\gamma_{0}, \gamma_{1}\right):(Y, \theta) \longrightarrow(X, \xi)$ be an arrow with $\left(\gamma_{0}, \gamma_{1}\right) ;\left(\pi_{0}^{i}, \pi_{1}^{i}\right)=\left(\phi_{0}^{i}, \phi_{1}^{i}\right)$ for all $i$. Then there is, for each $i$, a $Y \times X_{i}$ indexed family of isomorphisms $\left(y, x_{i}\right) ; \alpha_{i}:\left(y, x_{i}\right) ; \phi_{0}^{i} \longrightarrow\left(y, x_{i}\right) ; \gamma_{0}^{i}$ such that

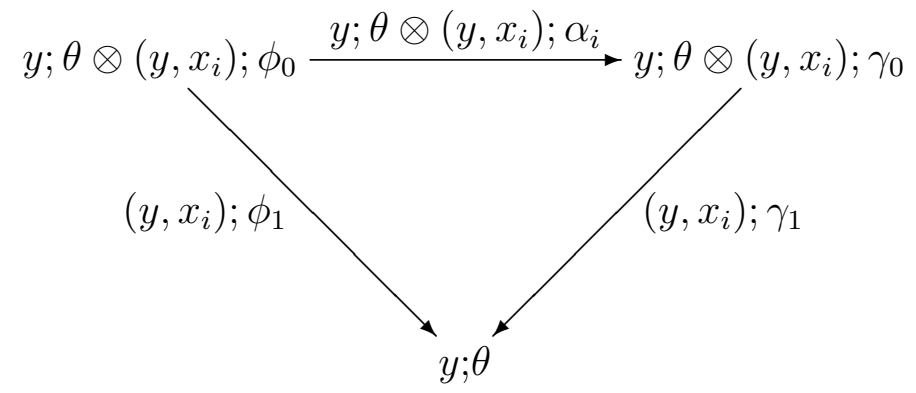


commutes. Let $\alpha: Y \times X \longrightarrow A_{1}$ be the unique family of isomorphisms whose restriction to $Y \times X_{i}$ is $\alpha_{i}$ for each $i$. Then one can readily see that these $\alpha$ define $\left(\phi_{0}, \phi_{1}\right)=$ $\left(\gamma_{0}, \gamma_{1}\right)$.

5.2 Cofree coalgebras. Since sums are products, the fact that $\otimes$ commutes with sums implies that it also commutes with products. Thus it follows immediately that the cofree cocommutative coalgebra on the object $(X, \xi)$ is given as

$$
G(X, \xi)=\prod_{n=0}^{\infty} S_{n}(X, \xi)
$$

with $S_{n}$ the $n$th symmetric tensor power, gotten by symmetrizing the $n$th tensor power. Note that $\mathbf{F u z}(\mathscr{A})$ does not have coequalizers in general, but it does have coequalizers of groups of automorphisms. In the present case, the carrier of the $n$th symmetric power is just the $n$th cartesian power symmetrized.

\section{References}

M. Barr (1979), *-Autonomous categories. Lecture Notes in Mathematics 752, Springer-Verlag, Berlin, Heidelberg, New York.

M. Barr (1991), *-Autonomous categories and linear logic. Math. Structures Comp. Sci. 1, 159-178.

R. Cockett and R. A. G. Seely (To appear), Weakly distributive categories. 